\title{
Deep Learning Based Remote Sensing using Convolutional Neural Networks
}

\author{
A. Shiny, Mrinmoy Kumar Das, Divyam Kumar Mishra, Manish Kumar Singh, Suman Maitra
}

\begin{abstract}
We describe our achievements in collecting alternating convergence points with a thickness of $7 \mu \mathrm{m}$ and focal lengths of 200 and $350 \mathrm{~mm}$, combined with shadow correction, deconvolution and significant neural frame training for transmission close to photography. Visual quality image. Although images taken using diffractive optics have been shown in previous papers, important neural structures have been used in the recovery phase. We use the imagery component of our imaging structure to activate the rise of ultralight cameras with remote identification for Nano and pico satellites, as well as small drones and solar-guided aircraft for aeronautical remote identification systems. . We extend the customizability of the liquid center focus on non-circular surfaces, forcing movement at the liquid convergence point of the surface. We study their trends and whether we can use them in optical structures.
\end{abstract}

Index Terms: deconvolution, neural framework, picosatellites, ultra-lightweight remote.

\section{INTRODUCTION}

In the proposed structure, we describe our progress in structure - a separate focus diffraction system with computerized distortion variations, which further contribute to the proposed procedure. Schuller began to study the single-point convergence system, where he proposed a special convergence chamber whose calculation deviated from the norm correction to prevent different convergence points of the structure.

Traditional imaging systems work from different optical components to eliminate anomalies. This complex arrangement allows clear and sharp images with amazing cost and optimum weight, and weight is a particularly basic standard for systems with high levels of long concentrates. The most outrageous capture is one of the structural barriers used in nano and pico satellites, near-space satellites (airborne) and small unmanned aerial vehicles. If in doubt, the near-space satellite logically releases the suppression of the payload, however, due to the most significant limitation of the auxiliary mass, the plane assembled under the sun can

Revised Version Manuscript Received on Jun 20, 2019.

A.Shiny, Department of CSE, SRM Institute of Science and Technology, Chennai, India.

Mrinmoy Kumar Das, Department of CSE, SRM Institute of Science and Technology, Chennai, India.

Divyam Kumar Mishra, Department of CSE, SRM Institute of Science and Technology, Chennai, India.

Manish Kumar Singh, Department of CSE, SRM Institute of Science and Technology, Chennai, India.

Suman Maitra, Department of CSE, SRM Institute of Science and Technology, Chennai, India. simply transfer most of the light weight of each wing. Since the sample must be fairly distributed along the contour of the aircraft, it attempts to connect the amazing convergence point with the large focus and sensor without the need for further support of the auxiliary section. Small drones require high-space lenses and ultra-light cameras to add intelligence.

We use the excitation parameter rectification linear unit (PReLU) instead of the rectifying linear unit (ReLU) from the first VDSR orchestra. ReLU covers negative responses, while PReLU parameters are negatively parameterized. This component of ReLU led to the approval of the "dead bend", which slowed down the plan and reduced execution. In addition, the proximity of PRELU's illegally obtained positive and negative answers provides an undisputed production technique for abusing information about entertainment pictures, where activation work is proposed to PReLU.

Our CNN is easy to show and provide ready-made choices. Preparing without a layout shows favorable results compared to the model that was previously prepared to solve the SR problem. We used different frames for each shader channel. The planning of the red channel due to self-assertion showed incredible performance, and when we started using the model that was capable of the red channel before, the blue channel was prepared to show the best performance.

\section{RELATED WORK}

\section{A. Deep Laplacian Pyramid Networks for Quick and Correct Super-Resolution}

In convolutional nervous systems, the onset of delayed deflection is good for a single super target. In this section, we describe the ultra-high resolution network of the Laplacian Pyramid (LapSRN) for legally reproducing sub-range fragments of images with high targets. At each level of the pyramid, our model uses map objects with rough targets, anticipating repeated high repetitions and using modified convolutions to improve test quality. Our system does not require cubic inclusion as a prepaid advance payment, so it can significantly reduce computational anomalies. We train the proposed LapSRN critical management, use reliable work to combat Char Bonnier, and achieve first-class entertainment. In addition, our architecture allows you to dynamically distribute multi-scale assumptions in a single direct connection because it supports resource-intensive applications. A broad tangible and abstract assessment of the reference data set indicates that the proposed invoice is firmly aligned with advanced 
methods in terms of speed and accuracy.

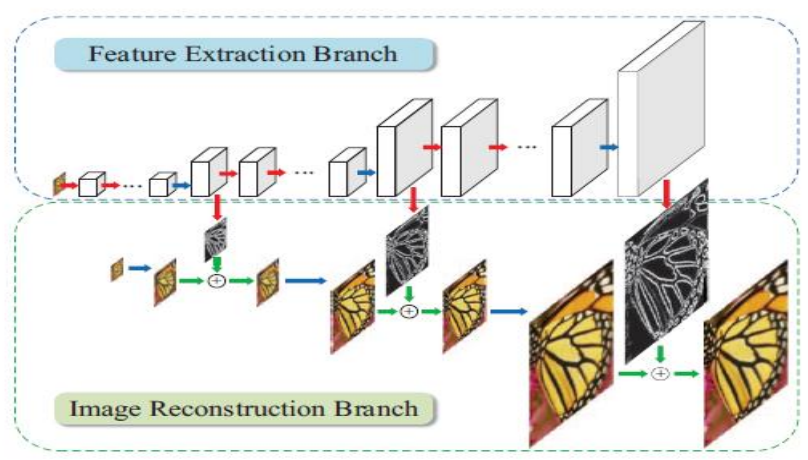

Fig. 1. Network architecture of LapSRN

\section{B. Hyperspectral Image Super Resolution by Transfer Learning}

The hyperspectral image of the super target is a very attractive topic in computer vision, which has attracted the attention of many analysts. Nonetheless, almost all current strategies indicate that observed hyperspectral images with low targets require different perceptions of similar scenes. This limits the use of super targets. In this paper, we propose a network structure that updates hyperspectral imaging targets by abusing typical image training: the connection between low/high targets is equivalent to the connection between low/high target hyperspectral images. In the proposed structure, a comparison of images with low and high targets can be studied by a deep convolutional nervous system, and a hyperspectral image can be replaced with a learning exchange. In addition, in order to consider the phantom trademarks in low-level and high-level hyperspectral images, a combined non-negative network factorization (CNMF) is proposed to achieve coordination efforts between low-level and high-level hyperspectral images, which leads to an estimated response concentration. Similar to the final member. Has a high spectral low level image. The findings of terrestrial and remote sensing information suggest that the proposed method performs a similar implementation without the need for any auxiliary images of similar scenes.

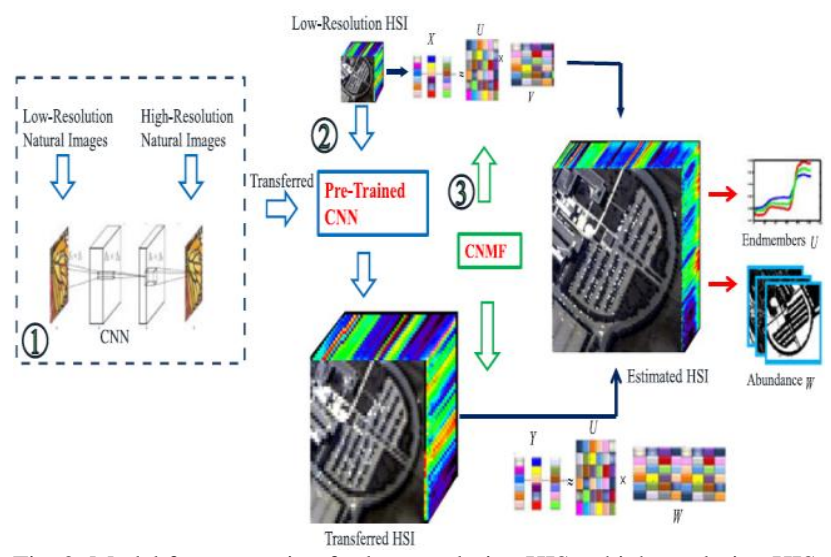

Fig. 2. Model for conversion for low resolution HIS to high resolution HIS

\section{Image Super-Resolution Using Deep Convolutional Networks}

We introduce an important training technique for a single Super Target (SR). Our program clearly checks the end-to-end mapping between images with low/high targets. Alignment is considered to be an important convolutional

neural structure $(\mathrm{CNN})$ that takes images with low target levels as data and gives images with high levels of target. Furthermore, we show that traditional SR strategies based on insufficient coding can be similarly considered to be

necessary convolution combinations. In any case, unlike the programs that are usually free to handle each part, our systems work together to improve all levels. Our important $\mathrm{CNN}$ has a lightweight structure, but it shows the highest quality of recovery and achieves a fast speed for proper online use. We explore various frameworks and parameter settings to achieve a trade-off between performance and speed. In addition, we extend our environment to adjust to three shader channels at the same time and often show the best distribution quality.

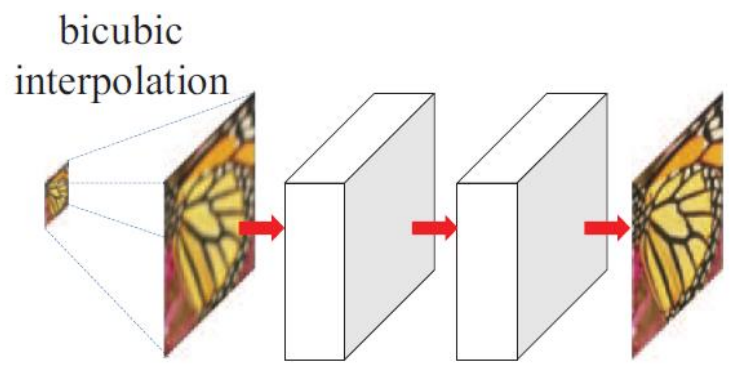

Fig. 3. Network architecture of SRCNN

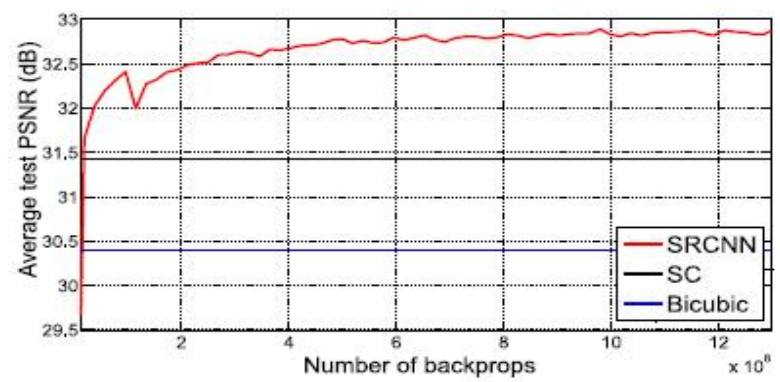

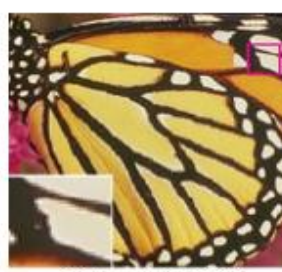

Original / PSNR

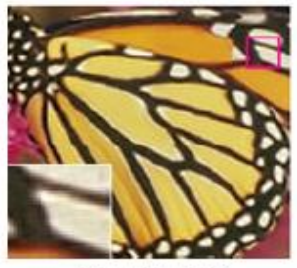

$\mathrm{SC} / 25.58 \mathrm{~dB}$

Fig. 4. Comparison of SRCNN with the sparse coding method on reconstruction of images

\section{Learning to Deblur}

We have described a learning-based approach to combat deconvolution of visually impaired images. It uses deep multi-level engineering, some of which is obtained as a result of ongoing neurological training work, some of which are incorporated into calculations that are explicitly used for image deconvolution. The framework is ready to begin to prepare for the preparation of various error preparations, which enables centralized execution during the deconvolution

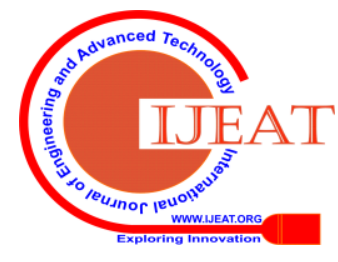


of visual impairments in terms of quality and execution time.

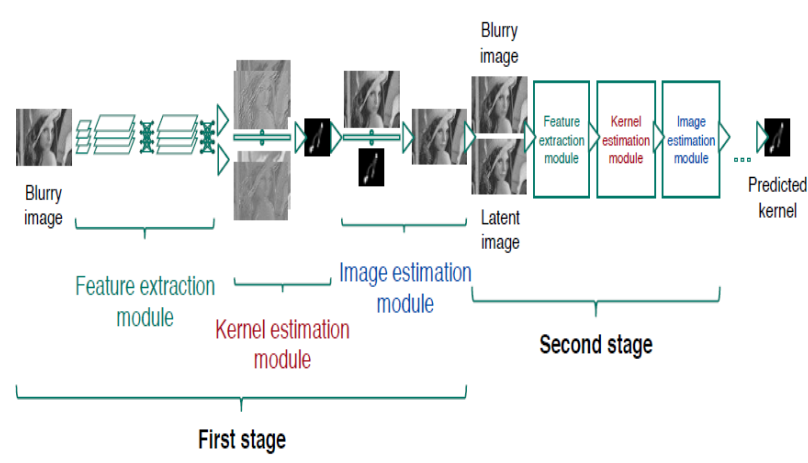

Fig. 5. Network architecture of Blind Deblurring Network

\section{E. Remote Sensing Scene Classification by Unattended Illustration Learning}

Due to the rapid development of innovative satellite sensors, remote sensing information with high spatial targets (HSR) has been widely used in military and non-military applications. In order to make full use of this information, remote sensing of the scene order becomes a mandatory and important reference point. This paper presents an unsupervised image training strategy for studying deconvolution systems for remotely detecting scene sequences. Initially, by limiting the playback error between the information image and the convolution result, a small scale deconvolution organization is used to gain mastery of the large number of maps and backlight channels for each image. The map of the formed elements can capture the edges of the image with high spatial targets and a large amount of data on the surface, which is undoubtedly necessary for remote image detection. From this point on, the Space Pyramid Performance (SPM) is used to summarize different proportions of bright areas to support the spatial design of HSR image scenes. A discrimination image of the HSR picture is obtained by combining the proposed weighted deconvolution model and SPM.

Finally, the image vector is the contribution of the help vector machine to complete the feature. We apply our method to two HSR image information collection tests: an information index for 21 scene categories and a Sydney information index for 7 land use categories. All of the test results performed using the proposed method exceeded most of the conditions for expressing human experience, indicating the feasibility of the proposed strategy.
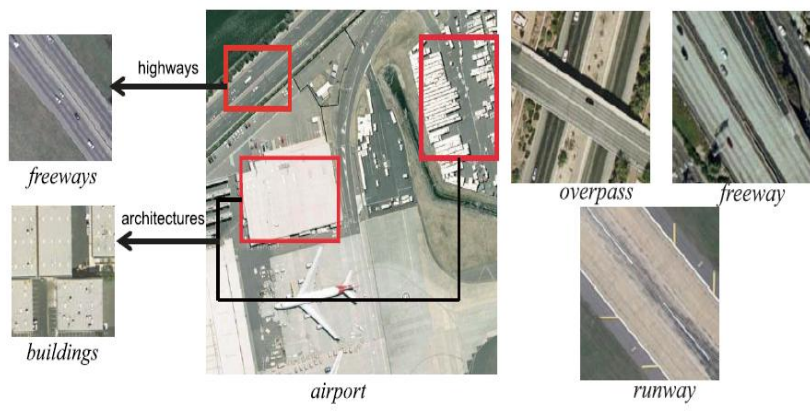

Fig. 6. Sample images for remote sensing scene

\section{PROPOSED APPROACH}

In the proposed structure, we demonstrate the progress in creating a single-lens diffractive structure using PC straightening variations to further develop the proposed method. Schuller began to study a framework with a single focus, where he proposed a separate camera for the focus of computational variation compensation to force out many frames with focus.

We use the startup parameter rectification linear unit (PReLU) instead of the rectification linear unit (ReLU) from the first VDSR scheme. ReLU inhibits side reactions, while PReLU's behavior in terms of negative mass is parameterized. This component of ReLU can lead to perceived "dead angles", reducing preparation speed and reducing execution. In addition, the proximity of the positive and near-reactions obtained by PReLU provides a gradual conservative approach to misuse of image reconstruction data, with work on the implementation of PReLU.

Our CNN is very detailed in terms of approval and preparation parameters. When prepared without preparation, this represents a preferred result compared to the beginning of the model previously prepared for the CP problem. We use a unique system for each coloring channel. Preparing the red channel from any load showed excellent performance, and when we started using the model previously prepared for the red channel, the blue channel was prepared to show the best performance.

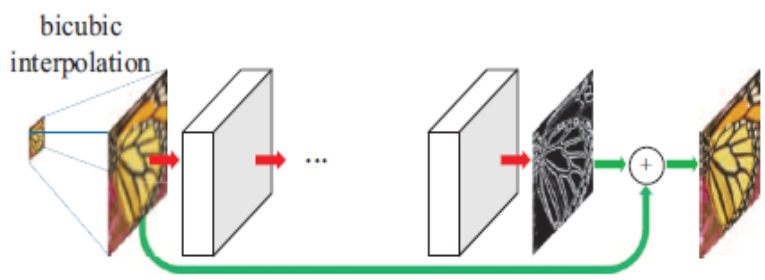

Fig. 7. Network architecture of VDSR

\section{A. Proposed System Advantages}

1) Computational updates result in $\mathrm{CNN}$ corrected images, replacing the usual deconvolution methods originally used.

2) The revision significantly improved image characteristics of emotions (using the human eye) and targets (by evaluating PSNR).

3) Additional advances in deconvolution can be achieved by reducing the noise estimated by the PSF.

4) In order to overcome the shortcomings of direct deconvolution mapping, we explored a deep learning image rework method that is effectively linked to SR due to a single image problem.

5) For SR problems, usually only the changes are applied to the brilliant segment, leaving the color segments unchanged.

\section{MODULES DESCRIPTION}

This section describes about the modules for our proposed system.

\section{A. Color Correction}

\section{Published By:}


In order to eliminate the shadow shift caused by the redistribution of vital forces between the diffractive forces (the difference between the captured shadow and the moderate deviation from the first shadow), the shadow tool must first be connected.

This ability of a single variable does not accurately indicate the connection between the unique and distorted tones in the entire shading space. These relationships are only in the coloring space.

\section{B. Chromatic Deblur by Non-blind Deconvolution}

The reward for chroma haze can be described as a non-blind deconvolution with a previously estimated PSF. For images taken using the diffractive focus, the green channel contains more shape data than the different channels. We propose a heuristic hypothesis to create the power of marking driven by the scientific expression of the central symphony. The main terminology includes two factors: a polynomial estimate of the main underlying elements and a hypothesis of delta work. Several autonomous factor forces in the polynomial are excluded to coordinate the information. As an estimate, we use a nonlinear least squares method with a strong recurrence of the smallest total residual.

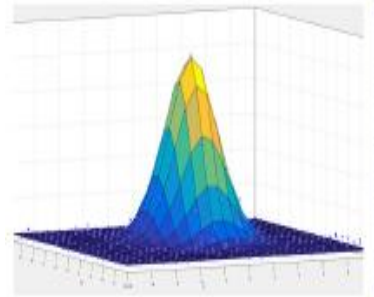

(a)

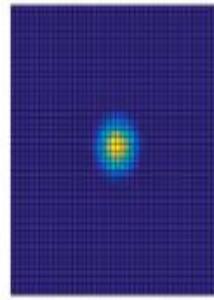

(b)

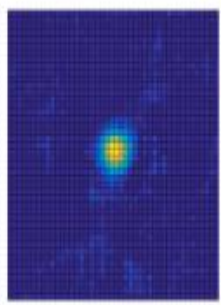

(c)
Fig. 8. (a) 3D view of PSF approximation of green colour channel. (b) Planar view. (c) Without approximation

\section{Deep Learning Based Reconstruction of Images and Removing of the Chromatic Blur}

To overcome the shortcomings of direct deconvolution, we explored a deep learning image transformation method that effectively links to SR from a single image problem. Images with low targets are scaled with bicubic injections and then processed with SRCNN and VDSR. The magnified image can be decoded as a non-linear neighborhood miss of the first image with a high target, while the NN output is recovered as a unique image.

Chromatic haze can also be decoded because the most recent target decreases with a certain scale factor. The territorial scope that is not regularly defined is no more than 2-4 pixels, which is equivalent to the reduction of the target, no more than 4 times.

\section{EXPERIMENTS AND RESULTS}

In this section, we consider the impact of the computational rework technique recommended in this paper on deconvolution-based regeneration techniques, as well as cross-channels from [3] and [2], as well as deconvolution and honing approach [1] and [5]. The PSNR aspect is used to evaluate the quality of the restored image.

\section{A. Examination with Current State of the Art}

We considered the nature of the results obtained using the deconvolution and honing procedures in [5] and the previous cross-channel deconvolution and the normalization of all differences in the L1 standard in [2]. According to the direct calculation in [4], we use the program we execute [2]. Table I shows the PSNR values for the adjustable system range. It independently contains the value of each channel as well as the value of the entire RGB image. Although [2] describes the large visual characteristics of the reconstructed image of the proposed colorless focus with contrast and quality published in [1] and [3], it is only for the convolutional mixture of multispectral PSF data. Like presenting a PSNR estimate. Rating Fresnel focus and colorless focus. These results were excluded from Table I due to the idea of the test suite being produced. Lines 1 and 2 in Table I show the effects [3] and [5] as templates, respectively. Line 3 shows that our symphony focus is significantly characterization of the rough image taken by the [5] diffraction focus, even exceeding the features of the reproduced image in [3].

\section{TABLE I. PSNR VALUES FOR CONTRASTING TYPES OF LENSES AND IMAGE RESTORATION METHODS}

\begin{tabular}{|c|c|c|c|c|c|}
\hline$\#$ & $\begin{array}{c}\text { Lens type and } \\
\text { reconstruction } \\
\text { method }\end{array}$ & \multicolumn{3}{|c|}{ Mean PSNR values for } \\
\cline { 3 - 6 } & $\begin{array}{c}\text { R } \\
\text { miffractive lens of } \\
\text { [5], raw images }\end{array}$ & $<8$ & $<13$ & $<12$ & G \\
\hline 2 & $\begin{array}{c}\text { Diffractive lens of } \\
\text { [3], using } \\
\text { deconvolution with } \\
\text { cross-channel prior }\end{array}$ & $<19$ & $<19$ & $<20$ & $<19$ \\
\hline 3 & Our raw images & 21.23 & 23.08 & 22.44 & 22.12 \\
\hline 4 & $\begin{array}{c}\text { Our raw images } \\
\text { with color correction } \\
\text { applied }\end{array}$ & 22.07 & 23.92 & 23.02 & 22.75 \\
\hline 5 & $\begin{array}{c}\text { Deconvolution with } \\
\text { raw PSF }\end{array}$ & 24.89 & 26.30 & 24.02 & 25.02 \\
\hline 6 & $\begin{array}{c}\text { Deconvolution with } \\
\text { approximated PSF }\end{array}$ & 25.45 & 27.91 & 27.21 & 26.45 \\
\hline 7 & $\begin{array}{c}\text { CNN filtering } \\
\text { applied to all } \\
\text { channels }\end{array}$ & 26.96 & 27.33 & 27.21 & 27.07 \\
\hline 8 & $\begin{array}{c}\text { CNN for R and B, } \\
\text { deconvolution with } \\
\text { approx. PSF in G } \\
\text { channel }\end{array}$ & 26.96 & 27.91 & 27.21 & 27.29 \\
\hline 9 & $\begin{array}{c}\text { Deconvolution and } \\
\text { sharpening from [5] }\end{array}$ & 25.76 & 27.85 & 26.18 & 26.15 \\
\hline 1 & $\begin{array}{c}\text { Deconvolution with } \\
\text { cross-channel prior }\end{array}$ & 26.03 & 27.85 & 26.27 & 26.33 \\
\hline
\end{tabular}

\section{B. Subjective Evaluation}

Fig. 9 shows captured and reproduced images viewed through a ground-based real image and our target map (captured outside). Board (a) and board (d1) - (d2) in the figure. Figure 9 shows the effect of a photo taken with our focus and then reproduced. Board (b) shows the result of remote image detection. Council (c) considers the visual characteristics of deconvolution based on CNN replication. Figure. Fig. 9 (c4) shows a pattern obtained by deconvolution of the intersecting channels earlier. 9 (c5) shows the meaning of our CNN-based approach. The deconvolution-based rendering contains boundaries within the window (see Figure 9 (c4)), which does not exist in the unique 
picture [see figure. 9(c3)]. This edge is an ancient mill that is suitable for any revolution based on deconvolution.

Although can be expected, CNN-based replication points to the outcome of peripheral free holidays. We conducted a series of open-air tests using a $300 \mathrm{~mm}$ focus and captured a number of different digital columns of target test patterns over a distance of $2 \mathrm{~km}$. The smallest rod that can be installed is $14 \mathrm{~cm}$ wide, as shown in Fig. 9(d3) - (d5). As you can see, the methods of deconvolution and $\mathrm{CNN}$ are common.

Our understanding of these results is the extended nature of the rough image produced by the consonant focus.

In addition, refuting past image change strategies and these new images requires different playback strategies.

Finally, the real image captured and reproduced on our platform has an average PSNR of $27 \mathrm{~dB}$, which is almost identical to that of a standard camera and mobile phone. This is the result of better focus mathematics and improved reproduction, currently including the deep nervous system.

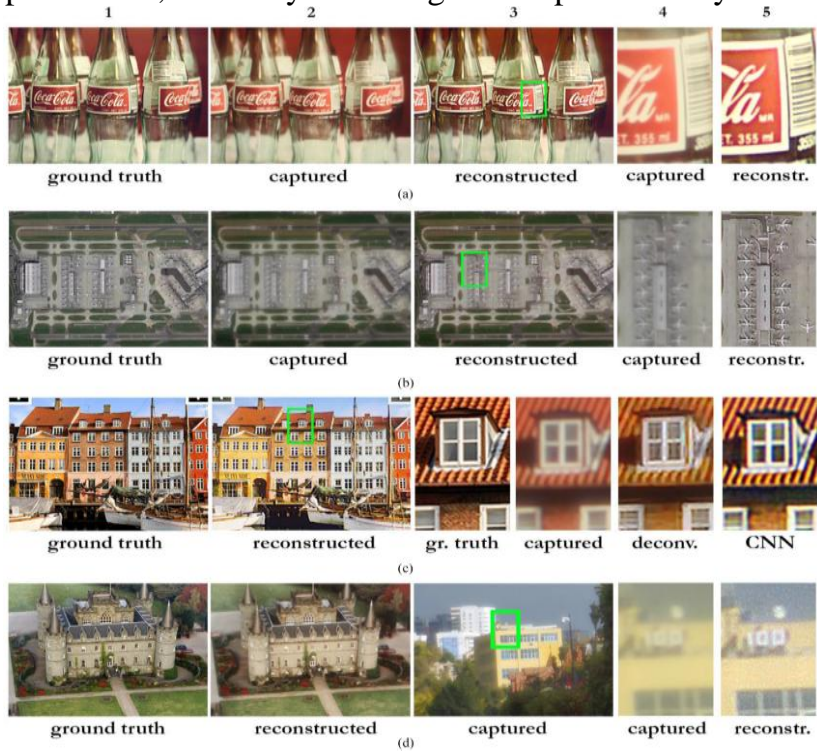

Fig. 9. Original images with comparison with the reconstructed ones

\section{CONCLUSION}

This paper describes the significant advances we have made in creating ultra-light focus based on diffractive optics. We added CNN to recreate the photos taken with our consonant techniques. We have also improved the process of making simfocal focus, resulting in significantly better, higher quality images contrasting with the fundamental diffraction focus while maintaining extremely low weight and thickness. In order to compensate for the symphony of the image focus, we propose to modify the image according to the combination of the depth convolution arrangement, and animate it by a single SR problem and image deconvolution. At the moment of connection to the images taken at our symphony focus, this strategy yielded a PSNR of $27 \mathrm{~dB}$, which is close to the traditional optical structure.

\section{REFERENCES}

1. A. Nikonorov, R. Skidanov, V. Fursov, M. Petrov, S. Bibikov, and Y. Yuzifovich, "Fresnel lens imaging with post-capture image processing," in Proc. IEEE Conf. Comput. Vis. Pattern Recog. Workshops, Boston, MA, USA, 2015, pp. 33-41.
2. Y. Peng, Q. Fu, F. Heide, and W. Heidrich, "The diffractive achromat full spectrum computational imaging with diffractive optics," in Proc. SIGGRAPH ASIA: Virtual Reality Meets Physical Reality: Model. Simulating Virtual Hum. Environ., 2016, Art. no. 4.

3. Y. Peng, Q. Fu, H. Amata, S. Su, F. Heide, and W. Heidrich, "Computational imaging using lightweight diffractive-refractive optics," Opt. Express, vol. 23, no. 24, pp. 31393-31407, 2015

4. A. Chambolle and T. Pock, "A first-order primal-dual algorithm for convex problems with applications to imaging," J. Math. Imaging Vis. vol. 40, pp. 120-145, 2011.

5. A. Nikonorov et al., "Comparative evaluation of deblurring techniques for fresnel lens computational imaging," in Proc. Int. Conf. Pattern Recog., Cancun, Mexico, 2016, pp. 775-780.

6. P. Atcheson, J. Domber, K. Whiteaker, J. A. Britten, S. N. Dixit, and B. Farmer, "MOIRE - Ground demonstration of a large aperture diffractive transmissive telescope," Proc. SPIE, vol. 9143, 2014, Art. no. 91431W.

7. C. Guo et al., "High-performance etching of multilevel phase-type Fresnel zone plates with large apertures," Opt. Commun., vol. 407, pp. 227-233, 2018.

8. C. J. Schuler, M. Hirsch, S. Harmeling, and B. Scholkopf, "Non-stationary " correction of optical aberrations," in Proc. Int. Conf. Comput. Vis., 2011, pp. 659-666.

9. F. Heide, M. Rouf, M. B. Hullin, B. Labitzke, W. Heidrich, and A. Kolb, "High-quality computational imaging through simple lenses," ACM Trans. Graph., vol. 32, no. 5, 2013, Art. no. 149.

\section{Authors Profile}

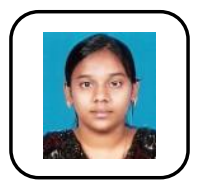

A.Shiny is currently a faculty advisor in SRM Institute of Science and Technology. Her current research interest is Network Security.

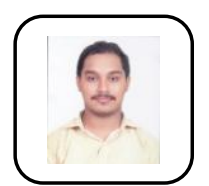

Mrinmoy Kumar Das is currently pursuing B.Tech (CSE) in his final year from SRM Institute of Science and Technology. He is currently working as an intern in Tata Consultancy Services in Chennai.

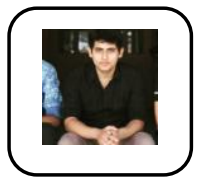

Divyam Kumar Mishra is currently pursuing B.Tech (CSE) in his final year from SRM Institute of Science and Technology. He is currently working as an IT consultant in Amtex Systems in New Delhi.

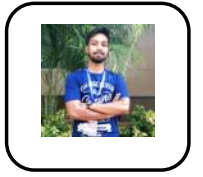

Manish Kumar Singh is currently pursuing B.Tech (CSE) in his final year from SRM Institute of Science and Technology. He is currently working as an intern in Steria Corporation in Chennai.

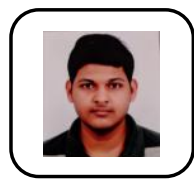

Suman Maitra is currently pursuing B.Tech (CSE) in his final year from SRM Institute of Science and Technology. He is currentlyworking as an intern in Temenos Pvt. Ltd. In Bengaluru. 